\title{
Existence, Uniqueness, and Approximation for Solutions of a Functional-integral Equation in $L^{p}$ Spaces
}

\author{
S. M. AFONSO ${ }^{1 *}$, J. S. AZEVEDO ${ }^{2}$, M. P. G. DA SILVA ${ }^{3}$ and A. M. ROCHA ${ }^{3}$
}

Received on December 10, 2018 / Accepted on February 15, 2019

ABSTRACT. In this work we consider the general functional-integral equation:

$$
y(t)=f\left(t, \int_{0}^{1} k(t, s) g(s, y(s)) d s\right), \quad t \in[0,1]
$$

and give conditions that guarantee existence and uniqueness of solution in $L^{p}([0,1])$, with $1<p<\infty$. We use Banach Fixed Point Theorem and employ the successive approximation method and Chebyshev quadrature for approximating the values of integrals. Finally, to illustrate the results of this work, we provide some numerical examples.

Keywords: functional-integral equations, $L^{p}$ spaces, existence, uniqueness, successive approximation.

\section{INTRODUCTION}

Nonlinear integral equations have been extensively studied in the literature, see for example integral equations of Urysohn type [6,7], Hammerstein type [3], and Volterra type [10], beyond the extension of these equations and applications (see [9] and references therein); the works cited had as a focal point conditions of existence of solution for such equations. In this sense, the theme has induced some authors to improve and extend these results to existence of solutions involving functional integral equations in the space $L^{1}([0,1])[2,4,5]$. For this reason, these authors have considered the equation:

$$
y(t)=f\left(t, r \int_{0}^{1} k(t, s) g(s, y(s)) d s\right), \quad t \in[0,1], r>0,
$$

\footnotetext{
*Corresponding author: Suzete M. Afonso - E-mail: s.afonso@unesp.br - https://orcid.org/0000-0003-3070-5856

${ }^{1}$ Universidade Estadual Paulista (UNESP), Instituto de Geociências e Ciências Exatas, 13506-900, Rio Claro-SP, Brazil E-mail: s.afonso@unesp.br

${ }^{2}$ Universidade Federal da Bahia - UFBA, Instituto de Ciências, Tecnologia e Inovação, Centro, 42802-721, CamaçariBA, Brazil - E-mail: jdazevedo@ufba.br

${ }^{3}$ Universidade Federal do Recôncavo da Bahia - UFRB, Centro de Ciências Exatas e Tecnológicas, Centro, 44380-000, Cruz das Almas-BA, Brazil - E-mail: mpinheiro@ufrb.edu.br, adson@ufrb.edu.br
} 
and proved the existence of solutions of that equation in $L^{1}([0,1])$.

In this way, they have concluded that equation (1.1) has a solution in this space. An extension of these results was given in $L^{p}([0,1]), p \geq 1$, by Karoui and Adel in [8], considering nonlinear integral equations of the Hammerstein and Volterra type. Moreover, in [11], the authors were able to guarantee the existence and uniqueness of the solution of Hammerstein integral equation in the $L^{p}([0,1])$ space. However, the results were limited to this specific type of equation. In order to fill this gap, we consider the functional-integral equation (1.1) and prove that, under certain hypotheses, it admits a unique solution in $L^{p}([0,1]), 1<p<\infty$. Here, we delete the term $r$ from our calculations.

As starting point, we show that, under certain conditions, the operator defined by the right hand side of (1.1) maps $L^{p}([0,1])$ into itself. It ensures that any solution of (1.1) lies in $L^{p}([0,1])$. And, under additional hypotheses, we prove that eq. (1.1) has a unique solution in $L^{p}([0,1])$, which can be obtained as the limit of successive approximations. The Chebyshev polynomial method [12] is used to solve the numerical integration due to high accuracy in small intervals and its simplicity. Numerical examples confirm the effectiveness of the proposed scheme.

The remainder of the paper is organized as follows. In Section 2 we present results on existence and uniqueness of solutions for functional-integral equation, considering the successive approximation method. In Section 3, we introduce the Chebyshev polynomials method and a recursive sequence to determine the solution of the non-linear system. Numerical examples are provided in Section 4.

\section{EXISTENCE AND UNIQUENESS}

In what follows, we assume that the function $f:[0,1] \times \mathbb{R} \rightarrow \mathbb{R}$ in (1.1) satisfies the Caratheodory conditions, that is,

i) $f(t, x)$ is continuous in $x$ for each fixed $t$;

ii) $f(t, x)$ is measurable in $t$ for each fixed $x$;

iii) there is a non-negative Lebesgue-integrable function $m:[0,1] \rightarrow \mathbb{R}$ such that $|f(t, x)| \leq$ $m(t)$, for all $(t, x) \in[0,1] \times \mathbb{R}$.

Theorem 1. Assume that the following conditions are satisfied:

(A1) There are a non-negative function $h_{1} \in L^{p}([0,1])$ and a non-negative constant $b_{1}$ such that

$$
|f(t, x)| \leq h_{1}(t)+b_{1}|x|^{q / p} \quad \text { for a.e. } t \text { in }[0,1], x \in \mathbb{R} \text {, and } \frac{1}{p}+\frac{1}{q}=1 .
$$

(A2) The kernel $k(t, \cdot)$ is measurable, belongs to the space $L^{q}([0,1])$ for all $t \in[0,1]$ and

$$
\left(\int_{0}^{1}|k(t, s)|^{q} d s\right)^{\frac{1}{q}} \leq M_{1}(t), \quad \text { for any } t \in[0,1],
$$


where $M_{1}$ is a non-negative function in $L^{p}([0,1])$.

(A3) The function $g(s, z)$ is a map from $[0,1] \times \mathbb{R}$ into $[0,1]$ satisfying Caratheodory conditions and such that

$$
|g(s, z)| \leq a_{0}(s)+b_{0}|z|
$$

where $a_{0}$ is a non-negative function in $L^{p}([0,1])$ and $b_{0}$ is a non-negative constant.

Under conditions $\left(A_{1}\right),\left(A_{2}\right)$, and $\left(A_{3}\right)$, the operator

$$
(A y)(t)=f\left(t, \int_{0}^{1} k(t, s) g(s, y(s)) d s\right), \quad t \in[0,1],
$$

is a map from $L^{p}([0,1])$ into $L^{p}([0,1])$.

\section{Proof.}

Firstly, note that $\|g(\cdot, y(\cdot))\|_{p}<\infty$ whenever $y \in L^{p}([0,1])$. Indeed, by Condition $(A 3)$, we have

$$
|g(s, y(s))|^{p} \leq\left(a_{0}(t)+b_{0}|y(s)|\right)^{p}, \quad \text { for all } s \in[0,1],
$$

and, therefore,

$$
\|g(\cdot, y(\cdot))\|_{p}=\left(\int_{0}^{1}|g(s, y(s))|^{p} d s\right)^{\frac{1}{p}} \leq\left(\int_{0}^{1}\left(a_{0}(s)+b_{0}|y(s)|\right)^{p} d s\right)^{\frac{1}{p}} .
$$

Using Minkowski’s inequality, we get

$$
\|g(\cdot, y(\cdot))\|_{p} \leq\left(\int_{0}^{1}\left|a_{0}(s)\right|^{p} d s\right)^{\frac{1}{p}}+\left(\int_{0}^{1} b_{0}^{p}|y(s)|^{p} d s\right)^{\frac{1}{p}}
$$

whence it follows that

$$
\|g(\cdot, y(\cdot))\|_{p} \leq\left\|a_{0}\right\|_{p}+b_{0}\|y\|_{p}<\infty .
$$

Now let us show that if $y \in L^{p}([0,1])$ then $A y \in L^{p}([0,1])$. In fact, for $y \in L^{p}([0,1])$ and $t \in[0,1]$, it follows from Condition $\left(A_{1}\right)$ that

$$
\begin{aligned}
|A y(t)|^{p} & =\left|f\left(t, \int_{0}^{1} k(t, s) g(s, y(s)) d s\right)\right|^{p} \\
& \leq\left[h_{1}(t)+b_{1}\left|\int_{0}^{1} k(t, s) g(s, y(s)) d s\right|^{q / p}\right]^{p} \\
& \leq\left(2 \max \left\{h_{1}(t), b_{1}\left|\int_{0}^{1} k(t, s) g(s, y(s)) d s\right|^{q / p}\right\}\right)^{p} \\
& =2^{p} \max \left\{\left[h_{1}(t)\right]^{p}, b_{1}^{p}\left|\int_{0}^{1} k(t, s) g(s, y(s)) d s\right|^{q}\right\} \\
& \leq 2^{p}\left(\left[h_{1}(t)\right]^{p}+b_{1}^{p}\left|\int_{0}^{1}\right| k(t, s) g(s, y(s))|d s|^{q}\right) .
\end{aligned}
$$


By Hölder's inequality, condition $\left(A_{2}\right)$, and eq. (2.3), we have

$$
\begin{aligned}
\left|\int_{0}^{1} k(t, s) g(s, y(s)) d s\right| & \leq \int_{0}^{1}|k(t, s) g(s, y(s))| d s \\
& \leq\left(\int_{0}^{1}|k(t, s)|^{q} d s\right)^{1 / q}\left(\int_{0}^{1}|g(s, y(s))|^{p} d s\right)^{\frac{1}{p}} \\
& \leq M_{1}(t)\left(\int_{0}^{1}|g(s, y(s))|^{p} d s\right)^{\frac{1}{p}} .
\end{aligned}
$$

For the sake of simplicity, denote $N_{1}(t)=M_{1}(t)\left(\int_{0}^{1}|g(s, y(s))|^{p} d s\right)^{\frac{1}{p}}$. Thus,

$$
\left|\int_{0}^{1} k(t, s) g(s, y(s)) d s\right|^{p} \leq\left[N_{1}(t)\right]^{p}
$$

and

$$
\begin{aligned}
|A y(t)|^{p} & \leq 2^{p}\left[h_{1}(t)\right]^{p}+2^{p} b_{1}^{p}\left|\int_{0}^{1} k(t, s) g(s, y(s)) d s\right|^{p} \\
& \leq 2^{p}\left[h_{1}(t)\right]^{p}+2^{p} b_{1}^{p}\left[N_{1}(t)\right]^{p} .
\end{aligned}
$$

Finally, as $N_{1} \in L^{p}([0,1])$, we obtain

$$
\int_{0}^{1}|A(y(t))|^{p} d t \leq 2^{p}\left\|h_{1}\right\|_{p}^{p}+2^{p} b_{1}^{p}\left\|N_{1}\right\|_{p}^{p}<\infty,
$$

which completes the proof.

Theorem 1 states that, under certain conditions, $A y \in L^{p}[0,1]$ if $y \in L^{p}[0,1]$. In this way, we look for solutions of integral equation $(1.1)$ in $L^{p}([0,1])$. Now, we would like to know which conditions are required for $f, k$ and $g$, in order to guarantee existence of solution this integral equation.

Theorem 2. Suppose that conditions (A1), (A2), and (A3) are satisfied. Furthermore, assume that:

(H1) the function $f:[0,1] \times \mathbb{R} \rightarrow \mathbb{R}$ satisfies Lipschitz condition in the second variable, that is, there is $M>0$ such that

$$
\left|f\left(t, x_{1}\right)-f\left(t, x_{2}\right)\right| \leq M\left|x_{1}-x_{2}\right|, \quad \text { for any } t \in[0,1] \text { and } x_{1}, x_{2} \in \mathbb{R} .
$$

(H2) the function $g:[0,1] \times \mathbb{R} \rightarrow \mathbb{R}$ satisfies Lipschitz condition in the second variable, that is, there is $L>0$ such that

$$
\left|g\left(s, z_{1}\right)-g\left(s, z_{2}\right)\right| \leq L\left|z_{1}-z_{2}\right|, \quad \text { for any } s \in[0,1] \text { and } z_{1}, z_{2} \in \mathbb{R} .
$$

Under such hypotheses, the successive approximation

$$
\left\{\begin{array}{l}
y_{0}(t)=0 \\
y_{n+1}(t)=f\left(t, \int_{0}^{1} k(t, s) g\left(s, y_{n}(s)\right) d s\right), \quad n=0,1,2,3, \ldots
\end{array}\right.
$$


converges almost everywhere to the exact solution of eq. (1.1) provided

$$
\int_{0}^{1} C^{p}\left[M_{1}(s)\right]^{p} d s:=N^{p}<1, \quad \text { where } C=M \cdot L .
$$

Therefore, eq. (1.1) has a unique solution in $L^{p}([0,1])$, which can be obtained as the limit of successive approximations.

\section{Proof.}

From Theorem 1 we have that the operator $A$ maps the space $L^{p}([0,1])$ into itself. Moreover, due to the assumptions on the function $f(t, x)$, the function values $(A y)(t)$ are well defined for all $y \in L^{p}([0,1])$. Using assumptions $(A 2),(H 1),(H 2)$, and Hölder's inequality, we obtain for $y^{1}, y^{2} \in L^{p}([0,1])$ and $t \in[0,1]$,

$$
\begin{aligned}
\left|A\left(y^{1}\right)(t)-A\left(y^{2}(t)\right)\right| & \leq M \int_{0}^{1}|k(t, s)|\left|g\left(s, y^{1}(s)\right)-g\left(s, y^{2}(s)\right)\right| d s \\
& \leq C \int_{0}^{1}|k(t, s)|\left|y^{1}(s)-y^{2}(s)\right| d s \\
& \leq C\left(\int_{0}^{1}|k(t, s)|^{q} d s\right)^{\frac{1}{q}}\left\|y^{1}-y^{2}\right\| \\
& \leq C M_{1}(t)\left\|y^{1}-y^{2}\right\|_{p},
\end{aligned}
$$

where $C=M \cdot L$. This implies

$$
\left\|A y^{1}-A y^{2}\right\|_{p} \leq N\left\|y^{1}-y^{2}\right\|_{p}
$$

with $N=\left(\int_{0}^{1} C^{p}\left[M_{1}(s)\right]^{p} d s\right)^{\frac{1}{p}}$. Due to condition $(2.5), A: L^{p}([0,1]) \rightarrow L^{p}([0,1])$ is a contractive map and Banach's fixed point theorem applies.

Consequently, under the hypotheses from Theorem 2, we already have the unique solvability of (1.1), where the method of successive approximation converges for starting point $y_{0} \equiv 0$, as we will show below.

For this method, we put $y_{0}(t)$ as the identically null function and successively

$$
y_{n+1}(t)=f\left(t, \int_{0}^{1} k(t, s) g\left(s, y_{n}(s)\right) d s\right), \quad n=0,1,2,3, \ldots
$$

Since $y_{0} \equiv 0$, it is easy to verify that $\left\|y_{1}\right\|_{p}<\infty$ (see Theorem 1$)$. 
Using Hölder's inequality, conditions (A1), (A2), (A3), (H1), and (H2), we obtain, for $n \geq 1$ and $t \in[0,1]$,

$$
\begin{aligned}
\left|y_{n+1}(t)-y_{n}(t)\right| & \leq M \int_{0}^{1}|k(t, s)|\left|g\left(s, y_{n}(s)\right)-g\left(s, y_{n-1}(s)\right)\right| d s \\
& \left.\leq C \int_{0}^{1}|k(t, s)| \mid y_{n}(s)-y_{n-1}(s)\right) \mid d s \\
& \left.\leq\left. C\left(\int_{0}^{1}|k(t, s)|^{q} d s\right)^{\frac{1}{q}} \cdot\left(\int_{0}^{1} \mid y_{n}(s)-y_{n-1}(s)\right)\right|^{p} d s\right)^{\frac{1}{p}} .
\end{aligned}
$$

Thus, for $t \in[0,1]$,

$$
\left.\left|y_{n+1}(t)-y_{n}(t)\right|^{p} \leq\left. C^{p}\left[M_{1}(t)\right]^{p}\left(\int_{0}^{1} \mid y_{n}(s)-y_{n-1}(s)\right)\right|^{p} d s\right) .
$$

Let $\mathscr{K}=\left\|y_{1}\right\|_{p}$. Inequality (2.8) implies

$$
\begin{gathered}
\left|y_{2}(t)-y_{1}(t)\right|^{p} \leq C^{p}\left[M_{1}(t)\right]^{p} \int_{0}^{1}\left|y_{1}(s)\right|^{p} d s=C^{p}\left[M_{1}(t)\right]^{p} \mathscr{K}^{p}, \\
\left|y_{3}(t)-y_{2}(t)\right|^{p} \leq C^{p}\left[M_{1}(t)\right]^{p} \int_{0}^{1} C^{p}\left[M_{1}(s)\right]^{p} \mathscr{K}^{p} d s \\
=C^{p}\left[M_{1}(t)\right]^{p} \mathscr{K}^{p} N^{p}
\end{gathered}
$$

and successively

$$
\left|y_{n+1}(t)-y_{n}(t)\right|^{p} \leq C^{p}\left[M_{1}(t)\right]^{p} \mathscr{K}^{p} N^{(n-1) p},
$$

which is equivalent to

$$
\left|y_{n+1}(t)-y_{n}(t)\right| \leq C M_{1}(t) \mathscr{K} N^{n-1}, \quad \text { for } n \geq 1, t \in[0,1] .
$$

Expression (2.9) shows that the sequence $\left(y_{n}(t)\right)_{t \in[0,1]}$ is a Cauchy sequence. Using this contractivity, we can verity that the series:

$$
\sum_{n=0}^{\infty}\left(y_{n+1}(t)-y_{n}(t)\right), \quad t \in[0,1]
$$

has the majorant

$$
C M_{1}(t) \mathscr{K}\left(1+N+N^{2}+\cdots+N^{j-1}+N^{j}+\cdots\right), \quad t \in[0,1] .
$$

Since this series converges on $L^{p}$-norm, the convergence of the sequence $\left(y_{n}(t)\right)$ to the (unique) solution of (1.1) is guaranteed by Banach Fixed Point Theorem [1, 13].

From Theorem 2, we have that the sequence $\left(y_{n}\right)$ in (2.4) converges to the exact solution since (2.5) holds. The following theorem establishes an estimative of the error generated by the successive approximation method of this sequence. 
Corollary 3. Assume that the conditions from Theorem 2 are satisfied. Then the sequence $\left(y_{n}\right)$ generated by the successive approximation method (2.4) satisfies the following inequality:

$$
\left\|y^{*}-y_{n}\right\|_{p} \leq \frac{N^{n}}{1-N}\left\|y_{1}\right\|_{p}
$$

where $y^{*}$ is the exact solution of (1.1).

Proof. The proof follows the same steps of Theorem 2 and therefore will be omitted here.

In the next section we will describe the methods used for discretization of the integral equation (1.1).

\section{DISCRETIZATION PROCEDURE}

In this section we apply Chebyshev polynomial method of the first kind (see [12]) to compute the integrals of one-dimensional functional-integral equation (1.1) and then we build a recursive sequence to solve the nonlinear system. Firstly, we start with some basic definitions.

Definition 1. Chebyshev polynomials of degree $n$ are defined as:

$$
T_{n}(x)= \begin{cases}\cos (n \arccos x), & \text { if }|x| \leq 1, \\ \cosh (n \operatorname{arcosh} x), & \text { if } x \geq 1 \\ (-1)^{n} \cosh (n \operatorname{arcosh}(-x)), & \text { if } x \leq-1\end{cases}
$$

In addition, these polynomials satisfy the following relations:

$$
T_{n}(\cos \theta)=\cos n \theta, \quad n=0,1,2, \ldots
$$

and

$$
\int_{-1}^{1} \frac{T_{n}(x) T_{m}(x) d x}{\sqrt{1-x^{2}}}= \begin{cases}0, & n \neq m \\ \pi, & n=m=0 \\ \frac{\pi}{2}, & n=m \neq 0\end{cases}
$$

Remark 1. The set of Chebyshev polynomials form an orthogonal basis in $L^{2}([0,1])$, so that a function $f \in L^{2}([0,1])$ can be approximated via expansion as follows:

$$
f(x) \approx \sum_{n=0}^{M} a_{n} T_{n}(2 x-2), \quad x \in[a, b]
$$

such that

$$
a_{n}=\frac{2}{\pi d_{k}} \int_{-1}^{+1} \frac{T_{n}(x) f\left(\frac{1}{2} x-\frac{1}{2}\right)}{\sqrt{1-x^{2}}} d x, \quad d_{k}= \begin{cases}2, & n=0 \\ 1, & n>1 .\end{cases}
$$


We estimate the unknown function $y(t)$ with the Chebyshev polynomials as

$$
y(t) \approx y_{M}(t)=\sum_{n=0}^{M} c_{n} T_{n}(t) .
$$

The unknown coefficients $c_{n}$ are determined by selecting collocation points $\left\{t_{i}\right\}_{i=0}^{M}$, where

$$
t_{i}=\frac{1}{2} x_{i}-\frac{1}{2}, \quad x_{i}=\cos \left(\frac{i \pi}{M}\right)
$$

The collocation method solves the nonlinear integral equation (1.1) using approximation (3.4) through the equations:

$$
y_{M}\left(t_{i}\right)=f\left(t_{i}, \int_{0}^{1} k\left(t_{i}, s\right) g\left(s, y_{M}(s)\right) d s\right) \quad 0 \leq i \leq M .
$$

Now, by substituting the expression (3.3) into (3.6), we get the following system:

$$
\sum_{n=0}^{M} c_{n} T_{n}\left(t_{i}\right)=f\left(t_{i}, \int_{0}^{1} k\left(t_{i}, s\right) g\left(s, \sum_{n=0}^{M} c_{n} T_{n}(s)\right) d s\right), \quad 0 \leq i \leq M,
$$

which in matrix form can be written in terms of the vector $c=\left[c_{0}, c_{1}, \ldots, c_{M}\right]^{T}$ as

$$
T c=F(c),
$$

with

$$
F(c)=\left[F_{0}(c), \ldots, F_{n}(c)\right]^{T},
$$

such that

$$
F_{j}(c)=\int_{0}^{1} k\left(t_{j}, s\right) g\left(s, \sum_{n=0}^{M} c_{n} T_{n}(s)\right) d x, \quad 0 \leq j \leq M,
$$

and

$$
T_{i, j}=T_{j}\left(t_{i}\right), \quad 0 \leq i, j \leq M .
$$

The solution to the system (3.7) is found by constructing recursively a sequence. In this way, for each iteration we solve a linear problem:

$$
c^{(k+1)}=T^{-1} F\left(c^{(k)}\right), \quad 0 \leq k<k_{\max },
$$

with $c^{(k)}=\left[c_{0}^{(k)}, c_{1}^{(k)}, \ldots, c_{M}^{(k)}\right]^{T}$. In addition to the stopping criterion $k>k_{\text {max }}$, the iterative process is carried out until:

$$
\left\|c^{(k+1)}-c^{(k)}\right\|_{2}<\text { tol } .
$$

Remark 2. We suppose to choose the number of integration points in such a way that the quadrature rule will not interfere with the successive approximation error, i.e, we assume the exact integration. 


\section{NUMERICAL EXAMPLES}

In this section, we describe some of the numerical experiments performed in solving the functional integral eq. (1.1), which can be treated by our Theorem 2 to illustrate the results of existence and uniqueness. For the numerical application, we use Picard iterative process and admit that the convergence is achieved when the stopping criterion has tolerance tol $=1 e-12$ in $L^{2}$-norm.

Here we employ the Chebyshev series and in the computation MATLAB package Chebpack available at the Mathworks website:

https ://www . mathworks . com/matlabcentral/fileexchange/32227-chebpack

as a stand-alone algorithm for solving nonlinear systems and investigating the performance of the numerical solution.

\section{EXAMPLE 1}

Consider the nonlinear functional integral equation:

$$
y(t)=\sin \left(\int_{0}^{1}(t-x)(y(x)) d x+(t-1) \cos (1)+\sin (1)\right), \quad t \in[0,1],
$$

with exact solution $y(t)=\sin (t)$. Take $k(t, x)=t-x$ and $f(t, z)=\sin ((t-1) \cos (1)+\sin (1)+z)$. It is easily verified in Theorem 2 that the hypotheses are valid for $1<p<\infty$. In this way, we have the guarantee of existence and uniqueness of the solution.

To establish the minimum number of integration points in terms of absolute errors, we note that, from 10 points of integration, we get the same convergence point with more or less iterations (see Fig. 1a). It allowed us to conclude that 10 points of integration are sufficient to preserve the convergence of the method. In the next experiment, we take 10 integration points and numerical solution putting $n=1,2,3,5$, and 10 iterations on the successive approximation method. The solutions are compared with the exact solution $y(t)=\sin (t)$ as described graphically in Fig. 1b. Already, Fig. 1c depicts the decay of the error on $L^{2}$-norm of the approximate solution considering a variation in the iterations number $n$, from 1 to 20 , while in Table 1 we present some values associated with these iterations. The results confirm the accuracy of the successive approximation method.

\section{EXAMPLE 2}

Consider the nonlinear functional integral equation:

$$
y(t)=\frac{1}{t+1} \log \left(\int_{0}^{1}(t x) \arctan (y(x)) d x-\frac{t}{3}+\exp (-t-1)\right)+\tan (t)+1,
$$

with $t \in[0,1]$ and exact solution $y(t)=\tan (t)$. Consider $k(t, x)=t x$ and

$$
f(t, z)=\frac{1}{t+1} \log \left(z-\frac{t}{3}+\exp (-t-1)\right)+\tan (t)+1 .
$$




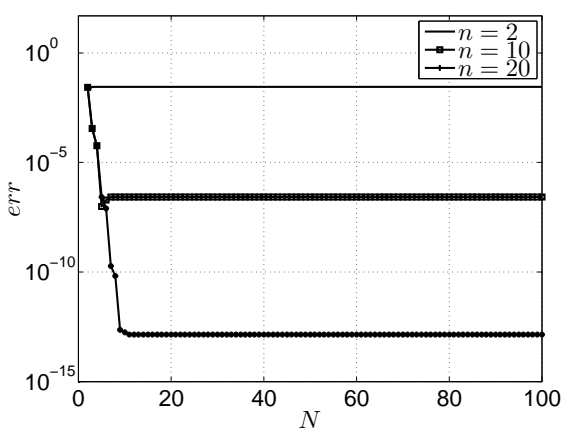

(a)

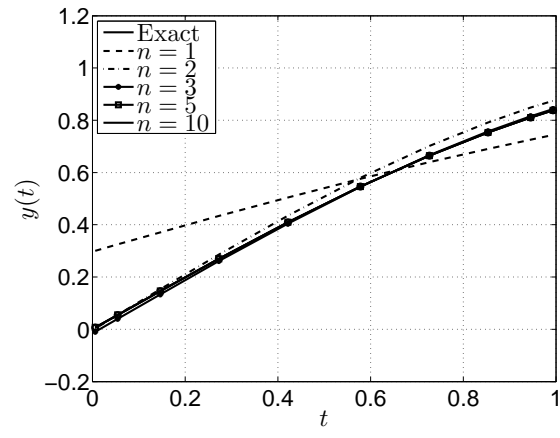

(b)

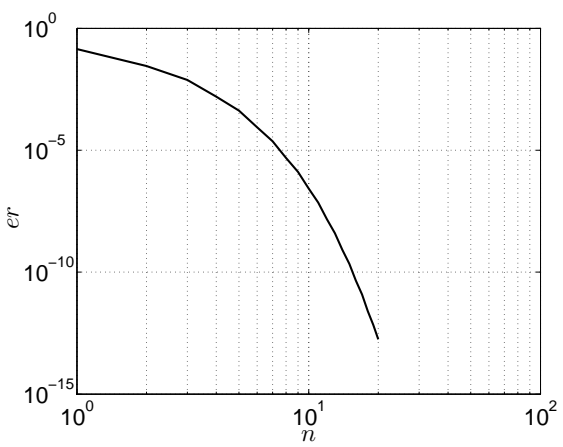

(c)

Figure 1: (a) Absolute error (in $L^{2}$-norm) of the numerical solution of eq. (4.2) in relation to number of integration points $N$ putting the iterations $n=2,10,20$, and using semi-log scale; (b) comparison of numerical solution, and exact solution, in 10 integration points; (c) absolute error (in $L^{2}$-norm) of the numerical solution of eq. (4.2) on iterations number $n$ from 1 to 20 using $\log$ - $\log$ scale.

Table 1: Error in the $L^{2}$-norm of the approximate solution with respect to eq. (4.1) for the iterations $n=1,2, \ldots, 20$.

\begin{tabular}{rrr}
\hline Iteration (n) & Error in the $L^{2}$-norm (err) & \\
\hline 1 & 0.139055224218022 & \\
2 & 0.28090214152532 & $\mathrm{e}-01$ \\
3 & 0.7514001013338 & $\mathrm{e}-02$ \\
4 & 0.1557774788458 & $\mathrm{e}-02$ \\
5 & 0.417391135550 & $\mathrm{e}-03$ \\
6 & 0.86414955296 & $\mathrm{e}-04$ \\
10 & 0.265922221 & $\mathrm{e}-06$ \\
12 & 0.14751583 & $\mathrm{e}-07$ \\
15 & 0.219260 & $\mathrm{e}-09$ \\
20 & 0.174 & $\mathrm{e}-12$ \\
\hline
\end{tabular}




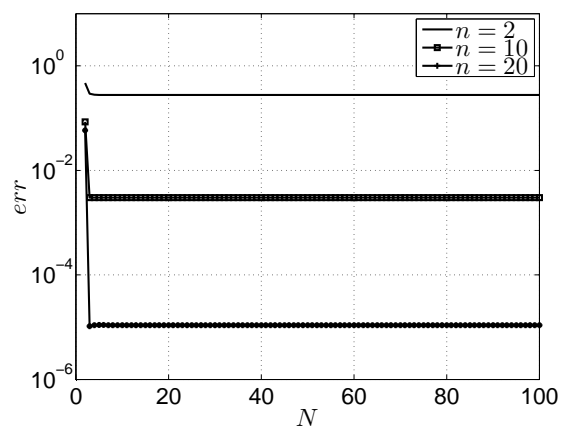

(a)

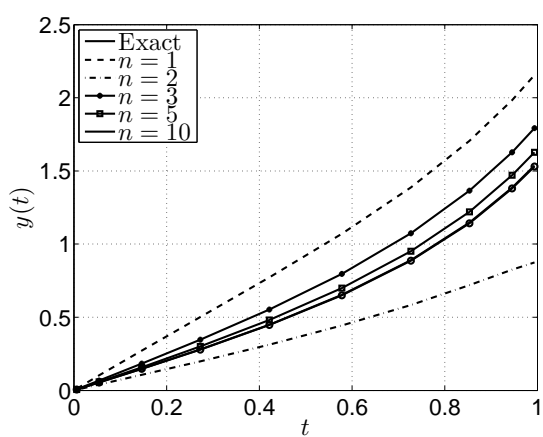

(b)

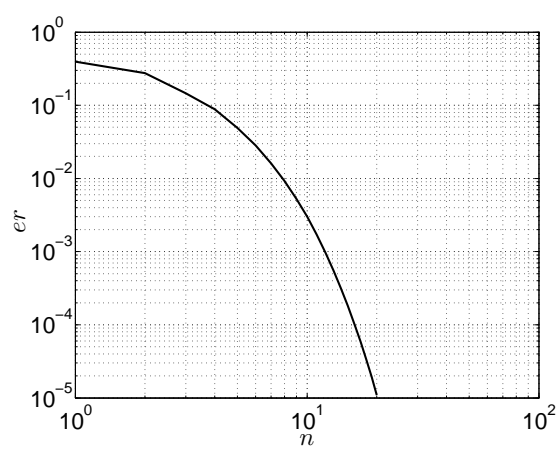

(c)

Figure 2: (a) Absolute error (in $L^{2}$-norm) of the numerical solution of eq. (4.2) in relation to number of integration points $N$ putting the iterations $n=2,10,20$, and using semi-log scale; (b) comparison of numerical solution, and exact solution, in 10 integration points; (c) absolute error (in $L^{2}$-norm) of the numerical solution of eq.(4.2) on iterations number $n$ from 1 to 20 using $\log -\log$ scale.

In this example, the hypotheses from Theorem 2 are also easily checked for $1<p<\infty$.

Similar to the previous experiment, in Figs. 2 we plot the approximate solutions of eq. (4.2) and the error associated with $L^{2}$-norm. The numerical solution has a good agreement with the exact solution. In Table 2 we exhibit again some numerical results of this error in $L^{2}$-norm.

\section{CONCLUSION}

In this paper we deals with the numerical approximation of nonlinear functional-integral equations, assuming the results in [5] as a starting point of the investigation. An important advantage of the method is that it does not require the specific transformations for nonlinear terms as required by some existing techniques. Furthermore, we can apply the method directly needing no linearization or perturbation. The method also converges to exact solution by successive approx- 
Table 2: Error in the $L^{2}$-norm of the approximate solution with respect to eq. (4.2) for the iterations $n=1,2, \ldots, 20$.

\begin{tabular}{rr}
\hline Iteration $(\mathrm{n})$ & Error in the $L^{2}$-norm (err) \\
\hline 1 & 0.394366600397979 \\
2 & 0.276308698116164 \\
3 & 0.146122318109685 \\
4 & $0.881455213962 \mathrm{e}-1$ \\
5 & $0.489253352732 \mathrm{e}-1$ \\
6 & $0.283969210188 \mathrm{e}-1$ \\
10 & $0.2994640228 \mathrm{e}-2$ \\
12 & $0.97457513 \mathrm{e}-3$ \\
15 & $0.18100617 \mathrm{e}-3$ \\
20 & $0.109484 \mathrm{e}-4$ \\
\hline
\end{tabular}

imations if such solution exists. Furthermore, we can use a few approximations for numerical purposes with a high degree of accuracy, when the conditions of existence and uniqueness of the results arising from this paper are respected. This is verified by the applications presented in the text.

RESUMO. Neste trabalho estabelecemos condições que garantem existência e unicidade de solução da equação integral-funcional geral

$$
y(t)=f\left(t, \int_{0}^{1} k(t, s) g(s, y(s)) d s\right), \quad t \in[0,1]
$$

em $L^{p}([0,1])$, com $1<p<\infty$. Utilizamos o Teorema de Ponto Fixo de Banach e aplicamos o método de aproximações sucessivas e a quadratura de Chebyshev para aproximar os valores das integrais. Finalmente, para ilustrar os resultados obtidos no trabalho, fornecemos alguns exemplos numéricos.

Palavras-chave: equações integrais-funcionais, espaços $L^{p}$, existência, unicidade, aproximações sucessivas.

\section{REFERENCES}

[1] K. Atkinson \& W. Han. "Theoretical Numerical Analysis: A Functional Analysis Framework". Texts Appl. Math. Springer New York (2001). URL https://books .google.com.br/books?id= C8iZGCKX JyMC.

[2] J. Banaś \& Z. Knap. Integrable solutions of a functional-integral equation. Rev. Mat. Univ. Complut. Madrid, 2(1) (1989), 31-38. doi:10.5209/rev_REMA.1989.v2.n1.18145. URL http://dx.doi. org/10.5209/rev_REMA.1989.v2.n1.18145.

[3] D.G. de Figueiredo \& C.P. Gupta. On the variational method for the existence of solutions of nonlinear equations of Hammerstein type. Proc. Amer. Math. Soc., 40 (1973), 470-476. doi:10.2307/2039394. URL http://www.jstor.org/stable/2039394. 
[4] G. Emmanuele. About the existence of integrable solutions of a functional-integral equation. Rev. Mat. Univ. Complut. Madrid, 4(1) (1991), 65-69. URL http://www . mat.ucm.es/serv/revmat/ vol4-1/vol4-1d.pdf.

[5] G. Emmanuele. Integrable solutions of a functional-integral equation. J. Integral Equations Appl., 4(1) (1992), 89-94. doi:10.1216/jiea/1181075668. URL http://projecteuclid.org/euclid.jiea/ 1181075668 .

[6] I.A. Ibrahim. On the existence of solutions of functional integral equation of Urysohn type. Comput. Math. Appl., 57(10) (2009), 1609-1614. doi:10.1016/j.camwa.2008.09.031. URL http://dx. doi. org $/ 10.1016 / j$. camwa. 2008.09.031.

[7] A. Karoui. On the existence of continuous solutions of nonlinear integral equations. Appl. Math. Lett., 18(3) (2005), 299-305. doi:10.1016/j.aml.2004.09.007. URL http://dx . doi .org/10.1016/ j.aml.2004.09.007.

[8] A. Karoui \& A. J. Existence and approximate $L^{p}$ and continuous solutions of nonlinear integral equations of the Hammerstein and Volterra types. Appl. Math. Comput., 216(7) (2010), 2077-2091. doi:10.1016/j.amc.2010.03.042. URL http://dx. doi.org/10.1016/j . amc. 2010.03.042.

[9] A.I. Košelev, M.A. Krasnosel'skij, T.O. Shapošnikova \& P. Zabrě̌ko. "Integral equations: a reference text". Monographs and textbooks on pure and applied mathematics. Noordhoff International Pub. (1975). URL https://books . google.com.br/books?id=vRSoAAAAIAAJ.

[10] M. Kwapisz. Bielecki's method, existence and uniqueness results for Volterra integral equations in $L^{p}$ space. J. Math. Anal. Appl., 154(2) (1991), 403-416. doi:10.1016/0022-247X(91)90046-3. URL http://dx.doi.org/10.1016/0022-247X (91)90046-3.

[11] M. Nadir \& B. Gagui. A numerical approximation for solutions of Hammerstein integral equations in $L^{p}$ spaces. São Paulo J. Math. Sci., 8(1) (2014), 23-31. URL https://www.ime.usp.br/ spjm/ articlepdf/494.pdf.

[12] R. Piessens \& M. Branders. Numerical solution of integral equations of mathematical physics, using Chebyshev polynomials. J. Comput. Phys, 21(2) (1976), 178 - 196. doi:https://doi.org/10. 1016/0021-9991(76)90010-3. URL http://www.sciencedirect.com/science/article/pii/ 0021999176900103.

[13] E. Zeidler. "Nonlinear Functional Analysis and its Applications I.(Fixed Point Theorems) 1986". Springer-Verlag, New York. 